\title{
Um programa de mentoria para estudantes de Medicina de uma universidade do Centro-Oeste brasileiro
}

\section{A mentoring program for medicine students at a University inthe Midwest of Brazil}

\author{
Patrícia Gonçalves de Moraes Brondani' (1) pgmoraes@bol.com.br \\ Adelair Helena dos Santos ${ }^{1}$ (D) adelair@ufg.br \\ Ana Maria de Oliveira' ${ }^{1}$ (D) dr.amoliveira@gmail.com \\ Carlos Eduardo Anunciação' (D) carlosgynufg@gmail.com \\ Marco Túlio Antônio Garcia-Zapata' (D) mctuliandip@gmail.com \\ Maria Amélia Dias Pereira1 (1) pereiramamelia@gmail.com
}

\begin{abstract}
RESUMO
Introdução: Neste artigo, fazemos um relato de experiência da implantação e do funcionamento de um programa de mentoria aplicado a estudantes de graduação do curso de Medicina de uma universidade do Centro-Oeste brasileiro. É consenso que a pressão dos cursos de Medicina provoca sobrecarga emocional e afeta negativamente os estudantes, e é preciso que medidas de apoio sejam implementadas.

Relato de experiência: O programa de mentoria da Faculdade de Medicina da Universidade Federal de Goiás teve um processo de construção lógico. Foi criado no início de 2015, pelo reconhecimento da necessidade da instituição em apoiar os acadêmicos durante sua graduação, fato evidenciado a partir do atendimento psicológico ao aluno e de uma pesquisa in loco. Os seguintes aspectos-chave caracterizam o programa: é oferecido como uma disciplina eletiva, pode ser feito até cinco vezes e,além de um encontro mensal com o mentor, o estudante escolhe as oficinas de que deseja participar, diversificando assim sua formação. Após 12 semestres de funcionamento, sintetizamos neste artigo uma parte de nossos resultados e algumas reflexões sobre o que queremos para o futuro do programa.
\end{abstract}

Discussão: A implementação da mentoria e suas adequações foram resultados de pesquisa, capacitação, discussões dos docentes e avaliação periódica pelos mentorados. As mudanças procuraram atender às expectativas e sugestões dos estudantes, objetivando atrair a atenção e satisfazer aos anseios dos discentes. Ao longo dos 12 semestres de funcionamento do programa, o interesse dos alunos pela matrícula na disciplina cresceu, com forte avaliação positiva, especialmente pela livre escolha e diversidade de oficinas, e pelo envolvimento do tutor.

Conclusão: As instituições de ensino devem estimular a criação de programas de mentoria. O programa relatado neste artigo tem tido boa aceitação por parte dos alunos e nos aponta bons resultados.

Palavras-chave: Tutoria; Estudantes de Medicina; Educação Médica; Saúde Mental.

\section{ABSTRACT}

Introduction: This article reports on the experience of implementing and running a mentoring program for medical school students at a Brazilian university in the Midwest region. It is widely accepted that the pressure at medical school is detrimental to the students and often leads to emotional overload. Therefore, supporting mechanisms must be implemented.

Experience report: The mentoring program at the Federal University of Goiás had a logical beginning. It was created in early 2015, after psychotherapy services offered to the students led to an in loco research study, which in turn pointed out the need to provide support to students throughout their undergraduate studies. There are some key characteristic aspects of the program: it's an elective course, which can be taken up to 5 times, and the students can choose the workshops they want to attend in addition to the monthly meeting with the mentor, which allows for diverse paths to be followed. After twelve semesters in existence, this article presents a summary of some of the results obtained and reflections on the future of this program.

Discussion: The implementation of the program and its adaptations resulted from research, professional development, discussions among professors and regular assessments by the mentees. Changes were made according to the expectations and suggestions of participating students, aiming to effectively meet their needs and represent a desirable course. Since its inception, the program has attracted increasingly more students, and enjoyed a strong positive evaluation, mainly due to the various workshops to choose from and engagement with the mentor.

Conclusion: Universities should support the creation of such mentoring programs. The program discussed in this article has been well received and points towards positive outcomes.

Keywords: Mentoring; Medicine Students; Medical Education; Mental Health.

\footnotetext{
1 Universidade Federal de Goiás, Goiânia, Goiás, Brasil.
}

Editora: Lia Marcia Cruz da Silveira

Recebido em 03/03/21; Aceito em 17/03/21.

Avaliado pelo processo de double blind review. 


\section{INTRODUÇÃO}

A formação do jovem médico é extensa no tempo e intensa nas experiências vivenciadas durante o curso', nas diferentes faculdades de Medicina no Brasil ${ }^{1-3}$ e no mundo ${ }^{4-8}$. A natureza da tarefa médica, as crises previsíveis de estresse causadas principalmente pelo choque entre o sonho e a realidade, e um contexto relacional marcado pela competitividade e aliado a questões sociais, financeiras e familiares tornam esse caminho, muitas vezes, solitário e permeado por sofrimento ${ }^{9,10}$.

No cenário nacional, as deficiências da educação básica ${ }^{11}$ e os problemas socioeconômicos do país têm resultado na formação de alunos com as seguintes características: deficiência em conteúdos, indisciplina, problemas na gestão dos estudos, dificuldades de acesso aos materiais didáticos, entre outras.

Agregado a essa realidade, o ensino superior passou nos últimos 14 anos por alterações significativas nos seus projetos pedagógicos de $\operatorname{cursos}^{12}$, período em que precisou enxugar cargas horárias e readequar conteúdos e disciplinas. Esse alinhamento atingiu em cheio os processos formais de ensino e a didática, com impactos relevantes tanto para os docentes quanto para os discentes dos cursos de graduação, em especial dos cursos de Medicina. $O$ resultado são alunos com visíveis dificuldades para atingir metas de desempenho acadêmico. Por conta disso, eles necessitam de criatividade para otimizar o próprio tempo e muitas vezes sacrificam suas demandas de lazer para obter melhor rendimento escolar. Isso tem resultado no avivamento da competição não salutar, especialmente fomentada pela baixa oferta de vagas de residência médica ${ }^{13}$

O somatório desses desafios torna o caminho do aluno de Medicina, muitas vezes, solitário e permeado por sofrimento. Para a maioria dos estudantes,constitui-se um grande desafio lidar com esse conjunto de experiências, de maneira que muitos podem apresentar queda em sua qualidade de vida ${ }^{14-16}$ e de seu desempenho acadêmico ${ }^{17}$, adoecimento psíquico e até mesmo redução de sua possibilidade de permanência na universidade, especialmente nos primeiros anos do curso ${ }^{18}$.

Em diversas faculdades de Medicina, programas de mentoria, em que um profissional experiente compartilha suas vivências e acompanha o jovem estudante ao longo do tempo, têm sido implementados. As experiências mostram que as atividades de mentoria ajudam os estudantes a superar os desafios enfrentados ${ }^{19}$. Nesse sentido, diferentes estratégias têm sido desenvolvidas pelas instituições de ensino médico para viabilizar e estimular a participação dos alunos e dos professores em programas de mentoria ${ }^{19}$.

\section{RELATO DE EXPERIÊNCIA}

\section{Cuidar do cuidador}

A preocupação com a saúde mental do estudante da Faculdade de Medicina da Universidade Federal de Goiás (FM/ UFG) sempre foi um desafio enfrentado pelos coordenadores do curso. Em 1998, por iniciativa do então diretor da FM/UFG, foi criado o Núcleo de Apoio ao Estudante de Medicina (Naem), cujo objetivo principal era oferecer uma escuta psíquica aos estudantes de Medicina que procuravam espontaneamente o programa em busca de ajuda. Os estudantes eram atendidos, orientados e, se necessário, medicados e encaminhados para acompanhamento especializado. Gradativamente, o programa se tornou mais conhecido, e foram incluídas outras práticas, como acolhimento aos calouros, palestras e seminários, ampliando-se a cultura de "cuidar do cuidador".

Em 2003, a Pró-Reitoria de Assuntos da Comunidade Universitária (Procom) da UFG, preocupada com a saúde mental de servidores e estudantes da instituição, criou o Programa Saudavelmente para promoção e assistência em saúde mental a todos os estudantes da universidade. Esse programa, ainda em pleno funcionamento, conta com equipe multidisciplinar e a participação de uma docente da Faculdade de Medicina, participante do Naem, como psiquiatra. Assim, o atendimento aos estudantes passou a ter caráter formal, em ambiente e horário definidos.

Apesar de as atividades do Naem terem sido incorporadas ao Programa Saudavelmente, a coordenação do Naem sempre considerou a necessidade de acompanhar de perto os estudantes de Medicina. Assim, elaborou-se um projeto de pesquisa no qual uma disciplina de Núcleo Livre denominada "Estratégias de Enfrentamento do Estresse Profissional" foi ofertada em 2011. Os objetivos dessa disciplina eram, por meio de uma pesquisaação, fazer uma escuta dos estudantes de Medicina sobre as adversidades do curso e oferecer ferramentas para que pudessem lidar melhor com o estresse e minimizar o sofrimento psíquico.

Em 2013, a disciplina foi ofertada novamente, atendendo 80 estudantes do curso de Medicina, divididos em duas turmas. Avaliouse a disciplina por meio de questionários que foram respondidos pelos participantes. Os resultados obtidos foram usados como base para uma pesquisa qualitativa que culminou com a tese de doutorado $O$ sofrimento psíquico na formação médica: percepções e enfrentamento do estresse por acadêmicos do curso de Medicina ${ }^{13}$. No momento da defesa da tese, a então diretora da FM/UFG, membro da banca, questionou qual era a proposta da doutoranda para contribuir com a saúde mental dos estudantes e reduzir o nível de sofrimento psíquico identificado pela pesquisa. Nesse momento, houve a proposta da implantação de um programa de mentoria, como atividade regular, com a participação de docentes do curso de Medicina atuando como mentores. 


\section{Um ambiente favorável}

No ano de 2014, a FM/UFG implantou o novo currículo, com conteúdo organizado em forma de módulos. Dentre as diretrizes, foi previsto um projeto de mentoria, pois a universidade percebeu que nesse momento havia uma conjunção de fatores que propiciariam a inserção dessa nova atividade. Entre os fatores, destacaram-se as discussões entre a coordenação do curso, os docentes e discentes acerca da implantação do novo currículo aliadas à identificação do sofrimento psíquico por parte dos alunos. Outros fatores determinantes para a implementação das atividades de mentoria foram o interesse da direção da FM/UFG em dar resposta às questões apresentadas pelos alunos, aliado à disponibilidade da professora de psiquiatria que desenvolvia o projeto de pesquisa sobre o comportamento psíquico dos estudantes de Medicina. Finalmente, destaca-se a proximidade e parceria do Programa Saudavelmente que, nesse momento, já se encontrava bem estruturado e com uma demanda crescente de estudantes.

As experiências mostraram que a capacitação de mentores e a adesão dos estudantes foram e continuam sendo os maiores desafios a serem enfrentados, pois o curso médico, com suas exigências, dificulta a participação de discentes e docentes nas atividades de mentoria. Assim, visando à preparação de professores que pudessem atuar como possíveis mentores e à apresentação aos estudantes das possibilidades dessa nova atividade, foi realizada em março de 2015, com apoio financeiro do Pró-Saúde, uma oficina de capacitação coordenada pela psicóloga Patrícia Bellodi da Faculdade de Medicina da Universidade de São Paulo (FMUSP). Durante os dois dias do encontro, apresentaram-se, para cerca de 50 professores da área de saúde da UFG, a experiência da FMUSP nos 16 anos do programa de mentoria, as atividades dos mentores e as características preconizadas. O Centro Acadêmico do curso de Medicina, que já estava mobilizado e interessado pelo tema de saúde mental, organizou a participação de cerca de 80 estudantes no evento, em que suas expectativas e necessidades foram ouvidas.

Nesse clima fértil, com apoio da direção da FM/UFG e da Pró-Reitoria de Graduação da UFG, decidiu-se pela oferta de mentoria em formato de disciplina na modalidade de Núcleo Livre. Dessa forma, no primeiro semestre de 2015, foram ofertadas 102 vagas para a disciplina, com a participação de 11 professores de diversas áreas da saúde que atuariam como mentores.

\section{Colocando ideias em prática}

Desde 2015, a disciplina "Mentoring na Faculdade de Medicina da UFG" é ofertada regularmente a cada semestre, para estudantes do curso de Medicina, na modalidade de
Núcleo Livre. São formados grupos com distribuição aleatória, compostos por até 12 estudantes de diferentes anos do curso, que podem cursar a disciplina por até cinco vezes. Os estudantes se encontram periodicamente com seus mentores, que são professores do curso de Medicina ou de diferentes cursos, ou ainda médicos que trabalham no Hospital das Clínicas da UFG na função de preceptores. Os mentores podem abordar temas previamente definidos ou aqueles sugeridos pelos estudantes no momento do encontro. Durante os encontros, realizamse uma escuta empática e a troca de experiências sobre as atividades e a vida dos estudantes. A disciplina tem carga horária de 16 horas, e, para aprovação, os estudantes devem ter no mínimo $75 \%$ de presença.

Além dos encontros mensais com os mentores, os acadêmicos participam de, no mínimo, quatro oficinas, que são ofertadas pela coordenação da disciplina e/ou da comunidade externa. As oficinas são escolhidas pelos estudantes conforme sua afinidade ou seu interesse. Esse projeto teve uma boa aceitação pelos estudantes do curso de Medicina, o que se comprova por seus relatos e pelo número crescente de matrículas desde o início do programa (Tabela 1).

\section{Ementa da disciplina}

Apresentam-se a seguira ementa e os objetivos da disciplina "Mentoring na FM/UFG".

Suporte pessoal e profissional durante o processo de formação; currículo médico; mercado de trabalho;

Tabela1. Número de participantes na disciplina de Mentoria, ofertada pela FM/UFG no período de 2015 a 2020

\begin{tabular}{cccc}
\hline Ano/semestre & $\begin{array}{c}\mathbf{N}^{\circ} \text { de } \\
\text { estudantes }\end{array}$ & $\begin{array}{c}\mathbf{N}^{\circ} \text { de } \\
\text { mentores }\end{array}$ & $\begin{array}{c}\mathbf{N}^{\circ} \text { de } \\
\text { oficinas }\end{array}$ \\
\hline $2015 / 1$ & 102 & 11 & 13 \\
$2015 / 2$ & 130 & 11 & 11 \\
$2016 / 1$ & 200 & 17 & 16 \\
$2016 / 2$ & 207 & 16 & 30 \\
$2017 / 1$ & 226 & 20 & 30 \\
$2017 / 2$ & 210 & 20 & 30 \\
$2018 / 1$ & 220 & 18 & 24 \\
$2018 / 2$ & 164 & 15 & 27 \\
$2019 / 1$ & 180 & 11 & 27 \\
$2019 / 2$ & 187 & 16 & 25 \\
$2020 / 3-$ ERE & 212 & 15 & 21 \\
$2020 / 1-$ ERE & 144 & 12 & 28 \\
$2020 / 2-$ ERE & 150 & 10 & Não definido \\
\hline TOTAL & 2.332 & 189 & 204 \\
\hline
\end{tabular}

*ERE - ensino remoto emergencial. 
estresse acadêmico; competição $x$ cooperação; qualidade de vida; rede social. Objetivo geral: Estabelecer, para grupos heterogêneos de alunos de diferentes anos, a figura de um mentor que os acompanhará ao longo do tempo durante o curso de graduação. Objetivos específicos: Criar um vínculo mais próximo e intenso entre professores $e$ alunos; promover a troca organizada de experiências entre o mentor e seus mentorandos, assim como entre os alunos de diferentes anos; acompanhar o desenvolvimento global dos alunos, incluindo seus conhecimentos, habilidades e atitudes, numa perspectiva humanística, ética e de compromisso social; identificar problemas no curso e na formação dos alunos, permitindo um encaminhamento mais imediato para a solução adequada; provocar uma formação integral do aluno de medicina e uma maior integração do curso a ele oferecido; oferecer oportunidade para conhecer diferentes técnicas para reflexão e melhora da qualidade de vida.

\section{Formato dos encontros}

Os encontros acontecem uma vez por mês, geralmente no período de fim de tarde, após o término das aulas, nas dependências da Faculdade de Medicina. O grupo é composto pelo mentor e por seu grupo designado de estudantes, e a duração do encontro é de uma a uma hora e meia. O aluno faz sua matrícula de maneira virtual por meio do Sistema Integrado de Gestão de Atividades Acadêmicas (Sigaa) da UFG e logo é incluído no grupo de WhatsApp da mentoria, em que passa a ter acesso às informações necessárias e se comunica com a secretária e com a coordenadora da disciplina. Os mentores previamente avisam qual a sua disponibilidade de dia da semana e horário dos encontros para aquele semestre. Os estudantes têm a opção de escolha do mentor ou do dia de sua preferência, o que, dentro do possível, é respeitado. A coordenadora da disciplina distribui os alunos matriculados tentando manter uma diversidade em relação às séries e monta os grupos de WhatsApp incluindo o mentor de cada grupo. A partir desse momento, a comunicação se estabelece entre mentoreados e mentores daquele semestre.

A dinâmica dos encontros varia entre os grupos, a depender das características individuais do mentor e também dos estudantes que os compõem. Alguns preferem estabelecer previamente o tema a ser discutido no próximo encontro, outros podem levar um convidado específico, outros podem escolher um filme para ser visto ou discutido. Algumas dinâmicas podem ser preparadas, como relaxamento. Geralmente há uma flexibilidade na escolha da semana em que ocorrerá o encontro, considerando períodos de provas dos alunos.

\section{Oficinas}

A ideia das oficinas surgiu como uma possibilidade de diversificar a formação dos estudantes de Medicina. Nos primeiros anos do programa, a maioria das oficinas oferecidas tinha uma parceria com o Programa Saudavelmente e ocorria em suas dependências. Por exemplo, tivemos oficina de meditação, yoga e automassagem. Depois estendemos para atividades oferecidas a toda a comunidade, como dança circular sagrada, roda de conversa sobre o autocuidado e as diversas lives durante a pandemia (saúde mental em tempos de pandemia, por exemplo).

Dois dos mentores já faziam um trabalho mensal com Constelação Familiar que desde 2015 faz parte da lista de oficinas oferecidas aos alunos da Mentoria. Esse é um exemplo de uma atividade aberta à comunidade e que, por ser considerada parte de uma disciplina, oferecia um estímulo extra para a participação dos mentoreados. Da mesma forma, outras atividades que poderiam levar a uma melhor qualidade de vida eram incluídas nas listas de oficinas oferecidas na mentoria, levando os estudantes de Medicina a ampliar seus recursos pessoais e ter acesso a práticas saudáveis. Nesse sentido, muitas parcerias foram criadas, e novas ideias, apresentadas. Uma das oficinas que caracterizam bastante a mentoria e da qual os alunos gostam bastante é a arteterapia. Acreditamos que diferentes abordagens podem servir de recursos para que o estudante de Medicina aumente sua resiliência e sua capacidade de enfrentamento das adversidades do curso. Dar a eles a oportunidade de conhecer essas abordagens é um grande caminho de ajuda.

Com o passar dos anos e com o grande número de alunos matriculados na disciplina, novas atividades abertas à comunidade passaram a ser consideradas como oficinas. Os próprios alunos nos apresentavam as novas propostas e perguntavam se elas poderiam se enquadrar como oficinas. Assim, várias foram incluídas, como o "Cine Clube da Medicina", a "Semana de Saúde Mental do Centro Acadêmico", o "Show do Esqueleto", apresentações artísticas etc. Basicamente, as atividades em que o tema fosse sobre saúde mental, qualidade de vida ou algo que agregasse à formação humanística dos estudantes eram consideradas como oficinas da mentoria e valiam como presença na disciplina. Essa diversificação de ofertas permitiu que os alunos conhecessem novas abordagens que favoreciam momentos de reflexão.

\section{Avaliações periódicas do programa}

A avaliação da disciplina é feita semestralmente pelos mentoreados, por meio de questionários, relatórios de oficina e depoimentos escritos. De maneira geral, os pupilos transparecem em seus relatos uma impressão positiva a respeito do programa e parecem se engajar nas atividades propostas de maneira satisfatória. Aspectos como oportunidade de conversar sobre suas dificuldades, espaço para troca de 
experiências, percepção da importância de se cuidar do cuidador, contribuição para o crescimento pessoal e ampliação do universo cultural são frequentemente destacados pelos mentoreados como aspectos positivos do programa. Quanto aos elementos que podem ser melhorados, os mentoreados costumam assinalar nas avaliações a necessidade de fornecer treinamento adequado aos mentores e a ampliação do número de encontros com seus mentores.

Com a experiência de 12 semestres da disciplina "Mentoring na FM/UFG", pudemos observar um interesse crescente dos alunos em matricular-se no programa, principalmente os estudantes de primeiro e segundo anos. A participação de alunos de anos mais avançados traz uma grande contribuição para os encontros, pois, por terem passado recentemente pelas experiências acadêmicas, podem auxiliar os novatos.

\section{Alunos facilitadores da mentoria}

Reconhecemos que uma forma de estimular a participação dos acadêmicos do ciclo profissionalizante é inseri-los num papel mais ativo na organização da mentoria. Em 2018, devido à procura dos próprios alunos que já haviam concluído os cinco semestres da disciplina e que manifestaram desejo de continuar a participar, foi criado um projeto de extensão (em parceria com a Faculdade de Medicina da Pontifícia Universidade Católica de Goiás - PUC Goiás), e passaram a ser oferecidas 36 vagas para capacitar alunos veteranos como facilitadores da mentoria. Como parte de suas atribuições, destacamos: ajudar a organizar, criar e divulgar atividades do programa, ajudar a monitorar as presenças das diversas oficinas oferecidas na disciplina e dar apoio aos mentores nos encontros mensais. Essa tem sido uma maneira de aumentar o engajamento dos acadêmicos na atividade e auxiliar o mentor em sua rotina burocrática. Observamos que esse projeto de extensão foi uma expansão natural do programa. No atual contexto de pandemia da coronavirus disease 2019 (Covid-19), com a mudança para modelo remoto, os alunos facilitadores perderam sua função, e o projeto de extensão ficou temporariamente comprometido.

\section{Expansão para outras faculdades locais}

Foi firmada em 2018 uma parceria com a Faculdade de Medicina da PUC Goiás, por meio do projeto de extensãocom o título de "Formação de Facilitadores para Mentoring na Área de Saúde". Sua finalidade foi expandir o projeto de mentoria da FM/UFG e compartilhar o conhecimento adquirido em três anos de experiência. Houve a participação de dois professores da Faculdade de Medicina da PUC Goiás e também de uma médica com experiência em arteterapia.

\section{Reajustes após o início da pandemia da Covid-19}

Em março de 2020, quando as aulas foram suspensas em razão da pandemia da Covid-19, a disciplina ainda não havia começado, porém os alunos já estavam matriculados, e os grupos, formados. Até julho de 2020, as aulas da UFG não haviam sido retomadas. Foi aberta a possibilidade de aulas remotas a partir desse momento, desde que $100 \%$ dos alunos matriculados concordassem com a mudança do formato. Nessa época, havia 160 estudantes de Medicina matriculados, e não foi possível obter a resposta de todos. Optou-se, então, pela oferta como disciplina de férias, no período de um mês, concentrando-se os quatro encontros e as quatro oficinas, de forma remota. Readaptou-se o programa da disciplina, e foram oferecidas 212 vagas. Os encontros remotos entre mentor e seus alunos ocorreram semanalmente, e as oficinas foram convertidas em programações on-line de diversas procedências, sugeridas pelos mentores e pelos próprios alunos, que ao final enviavam um relatório de participação. O primeiro semestre letivo de 2020 iniciou para toda a UFG no dia 29 de agosto, e nova turma de mentoring foi oferecida no modelo remoto, com as atividades concluídas em janeiro de 2021.

\section{DISCUSSÃO}

A instalação de um programa formal de mentoria na FM/UFG requereu dos docentes e da instituição esforços de capacitação e planejamento. Após um período de seis anos de experiência da oferta da disciplina, algumas considerações podem ser destacadas.

A mentoria na FM/UFG é oferecida na forma de disciplina, o que implica a possibilidade de geração de créditos ao currículo do aluno. Consideramos que esse aspecto pode enviesar as motivações dos alunos para se matricularem na disciplina. Debates e reflexões a respeito desse aspecto têm sido levantados entre os mentores, no desejo de otimizar a forma de inserção do programa na grade curricular do curso. Embora essa questão tenha sido foco de discussão, pouco material se encontra a respeito na literatura.

Os mentores do programa possuem liberdade de envolvimentos diferentes, com características e utilização de habilidades específicas, trazendo experiências diversificadas para os grupos. Consideramos que essa autonomia traz uma dinâmica acolhedora ao programa, garantindo liberdade para que o mentor atue conforme suas preferências. Desde o início desse projeto, vários mentores se voluntariaram a contribuir, e alguns estão atuantes desde o seu início. Muitos professores deixaram de participar, mesmo gostando da experiência, devido à sobrecarga da docência, principalmente neste momento de pandemia. Percebemos que a disposição mostrada pelo mentor na escuta durante os encontros valoriza 
a fala do estudante e estimula a postura de empatia e ajuda dos colegas. Os mentores podem ser modelos que influenciam no desenvolvimento pessoal e profissional dos alunos ${ }^{20}$, e é estimulado que, nos encontros, sejam trabalhadas questões envolvendo ética e profissionalismo, que complementam a formação acadêmica.

$\mathrm{Na}$ maioria das vezes, os temas dos encontros são trazidos pelos próprios estudantes e discutidos de forma aberta. Comumente, eles apresentam aquelas situações de sua vida acadêmica que geram maior sofrimento imediato, compartilham as dificuldades com provas, professores etc. A literatura mostra que esses são temas de maior interesse dos estudantes ${ }^{21}$. Uma ajuda importante vem, geralmente, dos próprios colegas, que, muitas vezes, já passaram pelas mesmas situações.

A mudança para mentoria no modelo remoto tem sido bem-sucedida até o momento em seus aspectos práticos. Os mentoreados mantêm o engajamento nas atividades, aparentemente sem dificuldades, talvez pelo fato de estarem familiarizados com novas tecnologias. Percebemos que a mentoria tem suprido uma demanda importante por interações afetivas nesta atual realidade da pandemia da Covid-19.

Reconhecemos que ainda há muitos desafios a serem enfrentados para manter o funcionamento do programa e sua oferta contínua de vagas, como: continuar a capacitação dos mentores, proporcionando ambiente acolhedor e empático para discutir sobre as dificuldades desse novo papel e sua consonância com as políticas educacionais da universidade; ampliar as redes de apoio aos estudantes para poder encaminhá-los quando necessário à mentoria ou aos serviços de atendimento social; identificar, apoiar e divulgar outras iniciativas de promoção social que existam na comunidade geral e participar delas, como forma de geração de vínculos; ampliar a participação em eventos nacionais e internacionais para troca de experiência com outros serviços e faculdades que ofereçam essa e outras formas de apoio aos estudantes de Medicina; catalogaras ações de apoio aos alunos oferecidas pela instituição e divulga-las aos estudantes de Medicina; estimular a cultura do autocuidado entre os acadêmicos, reconhecendo os estressores do curso e estimulando estratégias positivas de enfrentamento.

\section{CONCLUSÃO}

No atual cenário do ambiente educacional dos cursos universitários, ante as condições socioeconômicas da população, excepcionalmente nos cursos de Medicina, é importante desenvolver nas instituições programas de acolhida para os alunos. Um programa de mentoria institucionalizado estimula a formação de profissionais mais completos e com melhor preparo para o desempenho da atividade médica. As instituições devem fomentar sua criação e popularização, e estimular os docentes à participação e capacitação para essa atividade. Na FM/UFG, esse programa tem tido boa aceitação por parte dos alunos, o que nos motiva a acreditar que estamos na direção correta.

\section{CONTRIBUIÇÃO DOS AUTORES}

Adelair Helena dos Santos, Ana Maria de Oliveira, Carlos Eduardo Anunciação, Marco Túlio Antônio Garcia-Zapata e Maria Amélia Dias Pereira participaram da construção do artigo e também são mentores da FM/UFG. Patrícia Gonçalves de Moraes Brondani colaborou na elaboração do artigo.

\section{CONFLITO DE INTERESSES}

Declaramos não haver conflito de interesses.

\section{FINANCIAMENTO}

Declaramos não haver financiamento.

\section{REFERÊNCIAS}

1. Bellodi PL. Retaguarda Emocional para o Aluno de Medicina da Santa Casa de São Paulo (Repam): realizações e reflexões. Rev Bras Educ Med. 2007;31(1):5-14.

2. Fiorotti KP, Rossoni RR, Borges LH, Miranda AE. Transtornos mentais comuns entre os estudantes do curso de medicina: prevalência e fatores associados. J Bras Psiquiatr. 2010;59(1):17-23.

3. De Marco M. A face humana da medicina: do modelo biomédico ao modelo biopsicossocial. São Paulo: Casa do Psicólogo, 2003 pág 87-92.

4. Chan DW. Depressive symptoms and depressed mood among Chinese medical students in Hong Kong. Compr Psychiatry. 1991;32(2):170-80.

5. Maser B, Danilewitz M, Guérin E, Findlay L, Frank E. Medical student psychological distress and mental illness relative to the general population: aCanadian cross-sectional survey. Acad Med. 2019;94(11):1781-91.

6. Bonne O, Segman R, Katz M, Kaplan-DeNour A. Emotional distress in Israeli medical students. Harefuah. 2003;142(8-9):588-91, 647.

7. Hope V, Henderson M. Medical student depression, anxiety and distress outside North America: a systematic review. Med Educ. 2014;48(10):963-79.

8. Lee J, Graham AV. Students' perception of medical school stress and their evaluation of a wellness elective. Med Educ. 2001;35(7):652-9. doi: 10.1046/j.1365-2923.2001.00956.x.

9. Pereira MAD, Barbosa MA, de Rezende JC, Damiano RF. Medical student stress: an elective course as a possibility of help. BMC Res Notes. 2015;8(1):430.

10. Pereira MAD, Barbosa MA. Teaching strategies for coping with stress -the perceptions of medical students. BMC Med Educ. 2013;13(1):1-7.

11. Mendes MSS. Qualidade de ensino na escola pública: desafios e (im) possibilidades. Psicologia: Ensino \& Formação. 2010;1(2):61-71.

12. Salata A. Higher education in Brazil in the last years: reduction in inequalities of access? Tempo Soc. 2018;30(2):219-53.

13. Pereira MAD. O sofrimento psíquico na formação médica: percepções e enfrentamento do estresse por acadêmicos do curso de Medicina [tese]. Goiânia: Universidade Federal de Goiás; 2014 [acesso em 25 fev 2021]. Disponível em: https://bdtd.ibict.br/vufind/Record/UFG_ c19c7d65485ce829ea6effbd975029af.

14. Tempski P, Bellodi PL, Paro HB, Enns SC, Martins MA, Schraiber LB. What do medical students think about their quality of life? A qualitative study. BMC Med Educ. 2012;12(1):1-8. 
15. Paro HBMS, Morales NMO, Silva CHM, Rezende CHA, Pinto RMC, Morales $\mathrm{RR}$, et al. Health-related quality of life of medical students. Med Educ. 2010;44(3):227-35. doi: 10.1111/j.1365-2923.2009.03587.x.

16. Perotta B, Arantes-Costa FM, Enns SC, Figueiro-Filho EA, Paro H, Santos IS, et al. Sleepiness, sleep deprivation, quality of life, mental symptoms and perception of academic environment in medical students. BMC Med Educ. 2021;21(1):111.

17. Shawwa L Al, Abulaban AA, Abulaban AA, Merdad A, Baghlaf S, Algethami $A$, et al. Factors potentially influencing academic performance among medical students. Adv Med Educ Pract. 2015;6:65-75.

18. Arulampalam W, Naylor R, Smith J. Factors affecting the probability of first year medical student dropout in the UK: a logistic analysis for the intake cohorts of 1980-92. Med Educ. 2004;38(5):492-503. doi: 10.1046/j.13652929.2004.01815.x.
19. Bellodi P, Martins MA. Tutoria: mentoring na formação médica. São Paulo: Casa do Psicólogo; 2005 [acesso em 21 fev 2021]; Disponível em: https:// books.google.com.br/books?hl=pt-BR\&lr=\&id=fXgr24NDs5IC\&oi=fnd\&p $\mathrm{g}=$ PA5\&dq=Tutoria:+mentoring+na+formação+médica\&ots=ftVc-i6y54\& sig=9rLIdZDuz63tLESIxqR80dIR3sM.

20. Rose GL, Rukstalis MR, Schuckit MA. Informal mentoring between faculty and medical students. Acad Med. 2005;80(4):344-8.

21. Bellodi PL, Martinho T, Massaroppe B, Martins MA, dos Santos MAS. Temas para um programa de tutoria em Medicina: uma investigação das necessidades dos alunos da FMUSP. Rev Bras Educ Med. 2004;28(2):119-27. 